\section{The 35th Annual Meeting of the J.B. Johnston Club for Evolutionary Neuroscience and the 27th Annual Karger Workshop in Evolutionary Neuroscience}

The 2015 meetings of the J.B. Johnston Club for Evolutionary Neuroscience and Karger Workshop in Evolutionary Neuroscience will be held immediately before the annual meeting of the Society for Neuroscience on Thursday, October 15 (the Karger Workshop), and Friday, October 16 (the regular JBJC meeting). Both meetings will take place at the Congress Plaza Hotel, Chicago, Ill., USA.

This year's Karger Workshop, made possible by the continuing support of Karger Publishers, is organized by Alice Powers. It is titled 'The Role of Adult Neurogenesis in Plasticity: Evolutionary Insights'. The Workshop features eight invited talks by speakers who will review the relationship between neurogenesis and behavior. The speakers will report on a wide range of animal groups and technical approaches. On the following day, the program for the annual JBJC meeting will consist of 15 talks submitted by JBJC members selected by the JBJC Program Committee (Chris Braun, Bruce Carlson, and Naoyuki Yamamoto) plus a presentation by this year's invited Karger Speaker, Dr. Øyvind Øverli. The meeting also includes a 'data blitz' of very brief presentations. Additional information and the final schedule of talks will be mailed to JBJC members before the meeting and posted on the JBJC website (www.jbjclub.org).

\section{Karger Workshop in Evolutionary Neuroscience: The Role of Adult Neurogenesis in Plasticity: Evolutionary Insights}

Speakers giving presentations at the 2015 Karger Workshop in Evolutionary Neuroscience are listed below. The final schedule of talks will be sent to the membership prior to the meeting and will be available at the registration desk during the meeting.

- Barbara Beltz, Wellesley College, Wellesley, Mass., USA

- Kent Dunlap, Trinity College, Hartford, Conn., USA

- Alice Powers Stony Brook University, Stony Brook, N.Y., USA

- Lara LaDage, Penn State Altoona, Altoona, Pa., USA

- Carolyn Pytte, Queens College (CUNY), Queens, N.Y., USA

- Luca Bonfanti, University of Turin, Turin, Italy

- Hans-Peter Lipp, University of Zurich, Zurich, Switzerland, and KwaZulu-Natal University, Durban, South Africa

- Øyvind Øverli, Norwegian University of Life Sciences, Ås, Norway

\section{J.B. Johnston Club for Evolutionary Neuroscience Meeting Abstracts}

Abstracts for talks scheduled for the 2015 annual meeting of the J.B. Johnston Club for Evolutionary Neuroscience are listed in alphabetical order by presenting author. The final schedule of talks will be sent to the membership prior to the meeting and will be available at the registration desk during the meeting. This year's Karger Invited Guest will be Dr. Øyvind Øverli. The title of his talk will be: 'Reduced Neural Plasticity and Depression Like Behaviour after Stress: Pathology or Adaptation'?

\section{Brain Transcriptome Analysis of Alternative Reproductive Tactics in a Blenniid Fish}

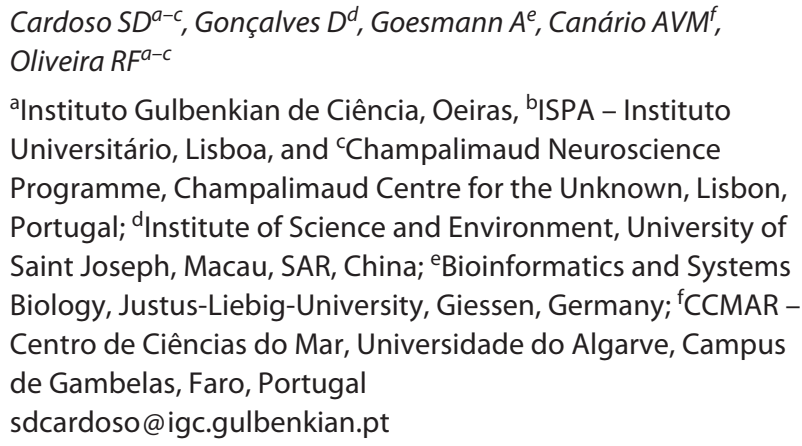

Organisms that share the same genotype can develop into different phenotypes depending on perceived environmental condition cues (aka phenotypic plasticity). Although not without its costs, phenotypic plasticity has been considered to facilitate adaptive evolution by allowing organisms to exploit a wider spectrum of resources when their fitness is reduced. Particularly in species with discrete variation in reproductive behavior, different life-history trajectories are observed that can either be fixed (e.g. genetic polymorphism), or plastic and therefore responsive to environmental factors (e.g. time of birth, resource availability) that will ultimately influence developmental processes. In the peacock blenny Salaria pavo males can follow either of two developmental pathways, grow directly into dominant nest-holder males or mimic the females' morphology and courtship displays in order to approach the nests of larger males to sneak fertilizations. These alternative reproductive tactics (ARTs) are sequential and consistent with body size (i.e. size at sexual maturity), such that sneaker males in the following breeding season switch to nest-holder males, go-

\section{KARGER 125}

C 2015 S. Karger AG, Basel

$0006-8977 / 15 / 0854-0287 \$ 39.50 / 0$ 
ing through a phase in which they are reproductively inactive (i.e. transitional males). In this work, we explored at the neurogenomic level how gene expression profiles differed between male morphs (i.e. nest-holder, sneaker and transitional males) and females. For this, RNA was isolated from whole brain tissue for each one of the four phenotypes, sequenced using Illumina HiSeq 2000 and de novo assembled. We found that at the brain level, expression of the plastic male tactic was accompanied by broader and divergent gene expression when compared to either females or nest-holder-males, both more similar between themselves. Overall, sneaker males differed from the other phenotypes in the expression of 643 unique transcripts $(78.38 \%$ annotated), followed by transitional males (597 transcripts; $75.71 \%$ annotated), females (562 transcripts; $76.51 \%$ annotated) and finally nest-holder males (517 transcripts; $78.92 \%$ annotated). Given the unique dichotomy present in sneaker males of the peacock blenny, behaviorally female-mimics but mature reproductive males, the lack of convergence between brain gene expression and behavior in sneakers to either females or nestholders points to a differential modulation of underlying proximate mechanisms of sexual behavior in sneakers.

\section{Active Electroreception in the Electric Eel (Electrophorus electricus)}

Catania KC

Department of Biological Sciences, Vanderbilt University, Nashville, Tenn., USA

ken.catania@vanderbilt.edu

Electric eels have fascinated naturalists and biologists for centuries because they have large electric organs that generate hundreds of volts of electricity when eels hunt or defend themselves. But how electric eels might have evolved was an enigma to Darwin because the function of small electric organs was unknown. In the 1950's Lissmann showed that weakly electric fish use small electric organs as part of an elaborate sensory system (active electroreception) providing the functional intermediate for the transition to electric weapons. Weakly electric fish subsequently became important model systems in neuroethological studies. However the active electrosensory system of the electric eel has been largely ignored. I conducted experiments using fast moving or stationary carbon conductors (while controlling for vision, mechanosensation, chemoreception, and biogenic electric fields) and show that electric eels can find, track, and attack conductors with great speed and accuracy. Electric eels also track fast moving prey below an agar barrier, under $940 \mathrm{~nm}$ infrared illumination. Details of these behaviors indicate that active electroreception is critical for eels to accurately guide strikes and suggest that eels depend heavily on both electrical weaponry and active electroreception when hunting.

\section{Retinal Sensitivity in Four Species of Frogs from Habitats with Different Light Regimes}

\author{
Farris $H^{a}$, Rosencrans $R^{a}$, Wisecarver ${ }^{a}$, Fellner $S^{a}$, Vumbaco $D^{a}$ \\ Gordon Wa, Richards-Zawacki $C^{b}$, Bazan Na \\ aNeuroscience Center, Louisiana State University Health \\ Sciences Center, New Orleans, La., and bepartment of \\ Ecology and Evolutionary Biology, Tulane University, \\ New Orleans, La., USA \\ hfarri@Isuhsc.edu
}

Anurans are models for research on the visual system. Surprisingly, although there is an extensive body of work detailing retinal architecture, development, physiology and behavioral feature detection, understanding of retinal sensitivity to functional stimuli under natural light conditions is still incomplete. This is especially true for comparisons between species which have evolved under different visual selective regimes. The overall goal of this project is to elucidate whether retinal specializations have evolved in response to the disparate visual ecology found across species, as terrestrial, arboreal and aquatic frogs may use vision under nocturnal and diurnal light regimes. First, using electroretinogram recordings, we measured retinal sensitivity in four species of frogs from different habitats: Xenopus laevis (aquatic, nocturnal); Hyla cinerea (arboreal, nocturnal); Rana pipiens (terrestrial, nocturnal) and Oophaga pumilio (terrestrial, diurnal). Following $12 \mathrm{~h}$ of dark adaptation, frogs' pupils were dilated and a series of broad spectrum (LED or Xenon strobe; Ganzfield dome), short duration flashes were presented in increasing intensity ( 0 to $\left.7.3 \mathrm{E}-4 \mathrm{~W} / \mathrm{cm}^{\wedge} 2\right)$ with increasing interflash intervals. Optical coherence tomography ensured retinas were healthy. Analysis of B-wave V/Log(I) curves showed that thresholds (light intensity required to elicit $10 \%$ re. max response) for $R$. pipiens and $H$. cinerea were significantly lower $\left(>1\right.$ order of magnitude $\left.\log \mathrm{cd} / \mathrm{m}^{2}\right)$ than those for both $X$. laevis and $O$. pumilio. These lower thresholds appeared sufficient for signal detection under the lowest natural ambient light conditions. Although, across all species, males had lower thresholds than females, within species comparisons were not significant. With respect to habitat, there was no systematic variation in $V / \log (\mathrm{I})$ slope (sensitivity) and saturation point. However, R. pipiens and $H$. cinerea did exhibit significantly shallower slopes than the other two species. These comparisons notwithstanding, measures of ERG slopes are useful in defining the range of light intensities for best processing of visual signals. Using the A-wave component of the ERG, we approximated the fractional circulating current in response to light flash. For isointensity stimuli, there was no significant evidence for habitat variance in amplification constant. However, isophoton measures must be tested. 


\section{What Makes a Head Turn? Vigilance Behavior Is Associated with Biomechanical Constraints and Retinal Configuration in Birds}

Fernandez-Juricic E, Shoemaker AL, Nelson MM, Moore BA

Department of Biological Sciences, Purdue University, West Lafayette, Ind., USA

efernan@purdue.edu

Vigilance behavior allows animals to gather visual information about potential threats (e.g., predators). Previous studies have shown that vigilance behavior patterns vary between species, but little is known about the factors affecting this degree of variation. We assessed the relationship between vigilance behavior (i.e., head movement rates) and the relative size of the centers of acute vision in the retina, taking into account body mass. We gathered information on 161 bird species. We found that head movement rates were affected by the interaction between body mass and the centers of acute vision, controlling for phylogenetic effects. Head movement rate decreased with body mass in a more pronounced way in species with smaller centers of acute vision compared to those species with larger centers of acute vision. These findings suggest that small bird species move their heads at a higher rate to scan the visual environment and compensate for the low proportion of the visual field with high visual resolution. In large bird species, the costs of moving the head may be higher and may be reduced by having retinas with a larger proportion of their visual field with high visual resolution. The overall implication is that vigilance behavior as an antipredator strategy may be the result of a trade-off between the biomechanical limitations of moving the visual system (head movements) and the retinal configuration.

\section{Neuroanatomical Evidence for Dopaminergic Modulation of the Peripheral Auditory System: Implications for Seasonal Auditory-Driven Social Behavior in a Vocal Fish}

Forlano PM

Biology, CUNY Brooklyn College and Graduate Center, Brooklyn, N.Y., USA

pforlano@brooklyn.cuny.edu

The plainfin midshipman fish, Porichthys notatus, has proven to be an excellent model to explore neural and hormonal mechanisms underlying vocal-acoustic communication in vertebrates. While steroid hormones are known to drive seasonal peripheral auditory plasticity in order to better encode the male's mate call, little is known of the neural substrates that underlie the motivation and coordinated behavioral response to acoustic social signals. Catecholamines, which include dopamine and noradrenaline, are good candidates for this function, as they are thought to modulate the salience of and reinforce appropriate behavior to socially relevant stimuli. We first characterized catecholaminergic (CA) innervation of the central and peripheral auditory system of midshipman and then tested if CA innervation of the auditory system is seasonally plastic and if CA neurons are activated in response to conspecific vocalizations. Neurobiotin backfills of the main auditory end organ, the saccule, combined with tyrosine hydroxylase immunoreactivity ( $\mathrm{TH}-\mathrm{ir})$, revealed a robust and direct $\mathrm{CA}$ innervation of both the peripheral and central auditory system. Dopaminergic neurons in the diencephalon (periventricular posterior tuberculum, TPp) are the source of TH-ir innervation of the saccule and the cholinergic octavolateralis efferent nucleus (OE), which also projects to the inner ear. These TPp neurons appear to have widespread projections to auditory nuclei, the spinal cord and the basal telencephalon and thus are in a unique position to influence sensory-motor integration and higher cognitive processes. Transmission electron microscopy combined with immunohistochemistry revealed that although punctate, vesicle-filled TH-ir swellings are found at the base of saccular hair cells in close proximity to afferent and efferent synapses, no other characteristics of synaptic specializations are observed, suggesting a prominent role for volume transmission of dopamine in the inner ear. In contrast, vesicle-filled $\mathrm{TH}$-ir profiles make direct inhibitory-like synapses on OE somata and axo-axonic contacts on inhibitory-like boutons, which in turn, synapse directly on somata of OE neurons. These results suggest potentially divergent and complex mechanisms of dopamine modulation of the inner ear, both directly and indirectly, via its central cholinergic efferent input. A seasonal analysis revealed that summer, reproductive females exhibited greater density of $\mathrm{TH}$-ir fibers in two forebrain auditory nuclei including the thalamus and greater density of TH-ir on somata and dendrites of the $\mathrm{OE}$. In contrast, winter, non-reproductive females had greater numbers of $\mathrm{TH}$-ir terminals in the saccule and greater $\mathrm{TH}$-ir fiber density in a region of the auditory hindbrain. Seasonal changes in dopamine innervation in both the inner ear and OE appear dependent on reproductive state in females and may ultimately function to modulate the sensitivity of the peripheral auditory system as an adaptation to the seasonally changing soundscape. Sound playback experiments revealed that males exposed to advertisement calls of other males exhibit a robust cFos-ir response in $\mathrm{TH}$-ir neurons in the TPp and noradrenergic locus coeruleus (LC). Activation of LC and TPp neurons may serve to coordinate appropriate behavioral responses to male competitors such as an increase in motivated vocal courtship. Overall, these studies support a role for catecholamines in seasonal auditory plasticity and auditory-driven social behavior in midshipman fish.

\section{Meta-Analysis of Ontogenetic Brain/Body Allometry across Mammals: Implications for Primate Encephalization and Fetal Growth Theories of Relative Brain Size}

Halley $A C$

University of California, Berkeley, Berkeley, Calif., USA

drewhalley@berkeley.edu

Relative brain size in any adult mammal is the endpoint of ontogenetic brain and body growth across pre- and postnatal development. Previous research in ontogenetic allometry has elucidated several important differences between mammalian clades, most notably that all primate species exhibit an evolutionarily novel de- 
gree of encephalization across fetal development when compared with other mammalian species. Prenatal allometry also offers a unique opportunity to test theories that implicate physiological constraints during gestation to explain species differences in relative brain size as adults; the maternal energy and placental invasiveness hypotheses predict preferentially faster fetal brain growth in species with relatively higher maternal metabolism and more invasive placentation, respectively. This meta-analysis of early ontogenetic allometry compiles data for twelve primate species representing platyrrhine and catarrhine clades (Callithrix jacchus, Cebus apella, Aotus azarae, Saimiri boliviensis, Saimiri sciureus, Trachypithecus cristatus, Macaca mulatta, Macaca nemestrina, Macaca radiata, Macaca fascicularis, Papio ssp., and Homo sapiens), as well as fourteen non-primate mammalian species representing diverse radiations (Mus musculus, Rattus rattus, Mesocricetus auratus, Oryctolagus cuniculus, Artibeus jamaicensis, Felis cattus, Canis familiaris, Sus scrofa, Ovis aries, Bos taurus, Stenella coeruleoalba, Phocoena phocoena, Macropus giganteus, Macropus eugenii). Contrary to a recent challenge to the theory of primate displacement, all primates appear to exhibit a shared trajectory of higher relative brain size across prenatal ontogeny as originally proposed. Neither relative BMR nor placental invasiveness correspond to higher slopes in prenatal allometric trajectories, as would be predicted from preferentially accelerated fetal brain growth. Evidence from aged growth data instead indicates that fetal brain growth rates are relatively constant across eutherian mammals following neurulation, and that somatic growth rate differences account for most variation in early developmental allometric growth. These results confirm that primate fetal allometric displacement is robust and shared among all species so far observed, and suggest that fetal brain growth is unconstrained by previously proposed mechanisms of nutrient delivery.

\section{Decreasing Sleep Requirement with Increasing Numbers of Neurons as a Driver for Bigger Brains and Bodies in Mammalian Evolution}

Herculano-Houzel S

Instituto de Ciências Biomédicas, UFRJ, Rio de Janeiro, Brazil suzanahh@gmail.com

Mammals sleep between 3 and $19 \mathrm{~h}$ per day, but what regulates daily sleep requirement is unknown. While mammalian evolution has been characterized by a tendency towards larger bodies and brains, sustaining larger bodies and brains requires increasing hours of feeding per day, which is incompatible with a large sleep requirement. Mammalian evolution, therefore, must involve mechanisms that tie increasing body and brain size to decreasing sleep requirements. Here I show that daily sleep requirement decreases across mammalian species and in rat postnatal development with a decreasing ratio between cortical neuronal density and surface area, which presumably causes sleep-inducing metabolites to accumulate more slowly in the parenchyma. Because addition of neurons to the non-primate cortex in mammalian evolution decreases this ratio, I propose that increasing numbers of cortical neurons led to a decreasing sleep requirement in evolution that allowed for more hours of feeding and an increase in body mass, which would then facilitate further increases in numbers of brain neurons through a larger caloric intake per hour. Coupling of increasing numbers of neurons to decreasing sleep requirement and increasing hours of feeding thus may have not only allowed but also driven the trend of increasing brain and body mass in mammalian evolution.

\section{Evolutionary Implications of Pinniped Vibrissal Innervation}

Mattson EE, Marshall CD

Department of Marine Biology, Texas A\&M University at

Galveston, Galveston, Tex., USA

mattsone@tamug.edu

Data on the origin of hair are few, and current theories are speculative. However, a popular theory is that hair arose to serve sensory, thermoregulatory, and protective functions. Vibrissae, or Follicle-Sinus Complexes (F-SCs), are specialized sensory hairs that detect vibrotactile cues and may have been the first type of hair to evolve. During their phylogeny, several mammalian lineages transitioned back to an aquatic environment, which likely placed strong selection pressures on vibrissal function and innervation. Innervation in terrestrial mammals appears conserved ( 150 axons/F-SC) but increased in certain lineages as they became more adapted to an aquatic lifestyle (semi-aquatic: $\sim 400$ axons/F-SC, fully aquatic: $\sim 1,400$ axons/F-SC). This innervation trend is observed in Orders Rodentia and Carnivora (particularly pinnipeds and mustelids), suggesting that vibrissal innervation may be independent of phylogeny. However, comparative data are few and prevent a complete comparison of innervation patterns among terrestrial, semi-aquatic, and fully aquatic species. Pinnipeds possess the greatest vibrissal innervation, but studies have not analyzed both macro- and microvibrissae. To address these data gaps, we investigated F-SC innervation in harp seals (Pagophilus groenlandicus) and included medialto-lateral analyses of mystacial vibrissae. We hypothesized that the F-SC innervation of harp seals is similar to that of other pinnipeds and that axon densities are consistent across the mystacial array despite F-SC size differences. The mean number of axons for harp seal macrovibrissae was $1,533.6 \pm 192.9$, a value consistent with other pinnipeds. Medial-to-lateral axon counts ranged from $550.3 \pm 97.4$ axons/F-SC to 1,632.1 \pm 173.2 axons/F-SC and averaged 1,221.2 \pm 422.3 axons/F-SC. These data suggest that conventional estimates using only macrovibrissae overestimate total innervation by $20 \%$ but still support the overall innervation trend seen in fully aquatic mammals. In our study, axon densities were consistent across the muzzle, but the distribution of axon bundles around the hair shaft varied from symmetrical in microvibrissae to asymmetrical in macrovibrissae. These results indicate that the apparent functional division of vibrissal regions observed during active touch experiments in pinnipeds may be due to variation in innervation distribution and hair shaft characteristics, not innervation investment. Our data are consistent with other pinniped innervation patterns and further support the hypothesis that vibrissal innervation investment increased as certain mammalian lineages became progressively more aquatic. 


\section{To Sting or Not to Sting? Intersexual Comparison in Stinging Behavior and Venom Effects in the Scorpion Species Centruroides vittatus}

Miller DW, Rowe M, Rowe A

Neuroscience Program, Michigan State University, East Lansing, Mich., USA

millerdy@msu.edu

There is a significant lack of knowledge concerning the behavior of scorpions, despite being the largest biomass in several ecologies and among the most ancient land animals One of the most recognizable features of a scorpion is their stinging 'tail', actually an extension of their body, used to inject venom into their targets for defensive, predatory, or reproductive purposes. However, venom injection comes at high metabolic cost, so scorpions do not necessarily sting at the slightest provocation, instead determining whether to sting based on evolutionary factors as well as the individual situation. To further probe these factors, the sexes of the North American species C. vittatus were compared in their stinging and venom-between the males and females of this species, there is a difference in their likelihood of stinging in defensive situations. Females will sting readily and several times, while males seemingly unwilling to sting, and will often stop after only one. This difference was quantified by electrically inducing the sensation of an attacking predator. Next, aspects of the venom injection were examined-how much the scorpions injected, and how many times they could inject venom before running out, finding no difference between the sexes in either of these. Finally, the pain induction effects of the venom on predators, mice in this case, were tested with a paw-licking assay for pain, and here it was seen that the males, less willing to sting a predator, had venom that caused more pain than that of the females when injected at the same volume and hydrated concentration. To put this in proper evolutionary context, however, the venom differences must also be examined on both their prey items and within the species for possible reproductive uses.

\section{Analysis of is/1-GFP, Tyrosine Hydroxylase (TH), GABA, and Parvalbumin in the Basal Ganglia of Adult Zebrafish Reveals Similarities to Tetrapod Situation}

Porter B, Stevens G, Mueller T

Division of Biology, Kansas State University, Manhattan, Kans., USA

bayleeporter@gmail.com

The zebrafish is an important genetic model organism to investigate the genetic and molecular-developmental factors underlying neurological disorders including Parkinson's Disease, autism spectrum, and stress and anxiety disorders. However, the field lacks a functional and comparative anatomical characterization of the zebrafish striatum to be able to extrapolate data to the mammalian and particular human situation. Such information would also greatly help to understand the genetic and developmental constraints that shape the evolution and function of the basal ganglia in non-tetrapod vertebrates. In this study, we shed light on the organization of the zebrafish basal ganglia by analyzing the distribution of isl1-GFP expressing neurons and other chemoarchitectonic and topological features of the teleostean telencephalon using immunohistochemistry on brain sections of adult zebrafish. To separate striatal from pallidal territories, for example, we analyze a combination of markers such as dopamine and GABA in a variety of transgenic line including $\operatorname{tg}($ isl1:GFP). Based on the location, cellular morphologies, and distribution of isl1:GFP expressing neurons, we hypothesize that subgroups of these neurons are homologous to striatal output neurons. In the future, we are going to test if some of these isl1-GFP expressing neuronal groups mediate motor behavior comparable to the mammalian striatonigral descending system.

\section{Predicting Visual Acuity from the Structure of Visual Cortex}

Srinivasan $\mathrm{S}^{a, b}$, Carlo $C N^{a}$, Stevens $C F^{a}$

asalk Institute for Biological Sciences, La Jolla, Calif., and

bUniversity of California, San Diego, Calif., USA

shyams@uci.edu

Vision researchers have shown that primates, especially diurnal primates, enjoy much better visual acuity than similar sized nonprimates. Comparative studies of the retina have shown that primates have more photoreceptors in the fovea, enabling higher resolution visual input much like modern digital cameras that improve image resolution by increasing the number of light detectors per pixel. The retinal image is mapped onto the primary visual cortex (V1), which performs computations integral to tests of visual acuity and our perception of the image. It is unclear, however, how primate V1s differ from non-primate V1s to enable better visual acuity? To answer this question, we compared visual cortices of primates and non-primates using stereology and theory. We found that $\mathrm{V} 1 \mathrm{~s}$ in primates are 2.5 times denser (more neurons per $\mathrm{mm}^{2}$ ) than non-primates. As primate V1s are denser, they have more neurons and thus more pinwheels - V1 structures that compute edge orientations - which improves computational power and visual acuity.

\section{The Hawk-Eyed Songbird}

Tyrrell LP, Fernández-Juricic $E$

Department of Biological Sciences, Purdue University, West Lafayette, Ind., USA

Ityrrell@purdue.edu

The vast majority of songbirds (Order Passeriformes) are insectivorous for at least part of the year. However, swallows are one of the only songbird groups that execute all aspects of hunting prey (search, detection, pursuit, and capture) in flight. We examined eye morphology and the retinal ganglion cell layer of wholemounted tree swallow (Tachycineta bicolor) retinas, cross-sectioned tree 
swallow foveae, and measured the dimensions of tree swallow visual fields using the opthalmoscopic reflex technique. We found that tree swallows, unlike other songbirds, have evolved a visual system similar to that of raptors (Orders Accipitriformes and Falconiformes). Like raptors, tree swallows have a bifoveate retina, unique among studied songbirds, that affords them a 'trident' of high quality vision with deep, central foveae directed laterally to either side of the head and shallow, temporal foveae directed forward. Tree swallows also have unusually long eyes that increase the focal length of the eye and thus the eye's spatial resolving power. Like raptors the tree swallow has an eye that is just as long as it is wide, whereas typical songbirds have an eye that is only two-thirds as long as it is wide. Raptors have unexpectedly narrow binocular fields $\left(20-39^{\circ}\right)$ but large blind areas behind the head $\left(60-101^{\circ}\right)$. Songbirds, on the other hand, have symmetrical binocular (average $40^{\circ}$ ) and blind areas (average $40^{\circ}$ ). Again, tree swallows fall out with the distantly related raptors with a blind area $\left(53^{\circ}\right)$ that is over twice the size of the binocular field $\left(23^{\circ}\right)$. As the tree swallow visual system demonstrates, the organization of sensory systems is not entirely constrained phylogenetically, but can be reshaped by the ecological challenges that particular species face.

\section{Similar Changes in Neuronal Circuitry Mediate Parallel Evolutionary Change in Sensory Processing of Communication Signals in Weakly Electric Fish}

Vélez $A^{a}$, Kohashi $T^{a, b}$, Carlson $B A^{a}$

aDepartment of Biology, Washington University in St. Louis, St. Louis, Mo., USA; bivision of Biological Science, Nagoya

University, Nagoya, Japan

avelezmelendez@wustl.edu

While several studies in neuroecology have investigated how natural selection shapes the gross anatomy of brain regions, we know little about the anatomical and physiological basis of evolutionary change in sensory processing at cellular and circuit levels. Using weakly electric fish of the family (Mormyridae), we investigated the neural basis for evolutionary change in sensory perception in a neural circuit devoted to communication behavior. Mormyrids communicate using pulse-type electric organ discharges (EODs) that are highly stereotyped and provide information about the identity of the sender. The ability to detect subtle variations in EOD waveform evolved independently in two mormyrid lineages. One lineage, known as 'clade A', consists of several species in the subfamily Mormyrinae; the other lineage belongs to the subfamily Petrocephalinae and is represented by only one known extant species: Petrocephalus microphthalmus. Interestingly, the perceptual ability to detect signal variation is associated with parallel changes in the gross anatomy of the central electrosensory system. The ancestral state of the midbrain exterolateral nucleus (EL) is small and undifferentiated. In both clade A and P. microphthalmus, however, the EL is enlarged and divided into separate anterior and posterior regions (ELa and ELp, respectively). To understand the underlying mechanisms of evolutionary change in sensory processing, we studied the anatomy and physiology of the neural circuitry in ELa/ ELp in four clade-A species and in P. microphthalmus, and the an- cestral EL circuit in Petrocephalus tenuicauda. We used neuronal tract tracing, immunohistochemistry, in vivo evoked potential recordings, and in vitro intracellular recordings.

In both lineages with an enlarged and differentiated ELa/ELp, ELa contained Small Cells and GABAergic Large Cells, while ELp contained Multipolar Cells. Large Cell projections were intrinsic to ELa, while Small Cells projected to ELp. Within ELa, incoming hindbrain axons followed long and convoluted paths suggestive of delay lines. In response to electrosensory stimulation with pulse trains, evoked potentials attenuated at short interpulse intervals in ELp, but not ELa, and intracellular recordings from Multipolar Cells in ELp revealed interval tuning. In $P$. tenuicauda, a species with a small and undifferentiated EL, we found Small Cells and Large Cells in the anterior end of EL. The Small Cells projected to the posterior end of EL, where we found Multipolar Cells. Incoming axonal projections from the hindbrain terminated predominantly in the anterior end of EL, but they did not follow a long and winding path as seen in ELa. Evoked potentials attenuated in response to pulse trains with short intervals in the posterior end of EL but not in the anterior end, and Multipolar Cells showed interval tuning.

Our results show that (i) the ancestral EL contains the building blocks for the ELa/ELp circuitry and (ii) similar evolutionary changes in this circuitry occurred in parallel in P. microphthalmus and clade A. We propose that the evolution of ELa/ELp, and the concomitant ability to detect EOD waveform variation, resulted from (i) a lengthening of incoming axons to form delay lines in the anterior part of EL, which allows for fine temporal analysis of EOD waveforms, and (ii) an overall increase in the number of cells within the anterior and posterior regions of EL to process a wider range of timing information. To the best of our knowledge, our results are the first to show how parallel changes in a neural circuit mediate parallel evolutionary changes in sensory perceptual abilities.

\section{Evolution of the Cerebellum in Light of the Expression of Zebrin II (Aldolase C) in the Cerebellum of Mammals, Birds, and Non-Avian Reptiles}

Wylie DR ${ }^{a}$, Aspden Ja, Gutierrez-Ibanez $C^{b}$, Iwaniuk $A N^{c}$, Hoops $D^{d}$

aNeuroscience and Mental Health Institute, University of Alberta, Edmonton, Alta., Canada; 'behrstuhl für Zoologie, Technische Universität München, Freising-Weihenstephan, Germany; 'Department of Neuroscience, University of Lethbridge, Lethbridge, Alta., Canada; dUniversity Research School of Biology, Australian National University, Acton, A.C.T., Australia dwylie@ualberta.ca

In both birds and mammals, the cerebellum is highly developed convoluted structure, consisting of numerous transverse lobules. The fundamental organization of the cerebellum, however, consisted of sagittal zones that are orthogonal to the lobules. This is apparent in the pattern of afferent inputs to the cerebellum, projection patterns of Purkinje cells (PCs), and PC response properties. The expression of several molecular markers is also parasagittally organized. The most thoroughly studied of these is zebrin II (ZII). In both birds and mammals, ZII is expressed in PCs 
such that there are sagittal stripes of high expression (ZII+) interdigitated with stripes of little expression (ZII-). In mammals, this alternating pattern of ZII+/ZII- stripes occurs in lobules I-V, and VIII-IX of the vermis, but in lobules VI-VII and X, the striping pattern is absent, as almost all PCs in these lobules are ZII+. In birds, the pattern of ZII expression in the cerebellar vermis is essentially identical. The exception is lobule I, which has alternating $\mathrm{ZII+/}$ - stripes in mammals, but in birds all the PCs are ZII+ve. This suggests that the pattern of ZII expression is highly conserved, and perhaps present in extant reptiles. In this study we examined ZII expression in a snake, the Western diamondback rattlesnake (Crotalus atrox), and a lizard, the central-netted dragon (Ctenophorus nuchalis). In snakes, the cerebellum is small, consisting of a single unfoliated sheet overlying part of the medulla. Within this small cerebellar cortex of the rattlesnake, ZII was not expressed as a series of alternating ZII+ and ZII- stripes rather all PCs were ZII+ve. In a previous report it has been shown that all Purkinje the cerebellum in turtles are ZII+ve. In lizards, the cerebellum is relatively larger compared to snakes, but is not foliated. It consists of a single sheet that folds back onto itself such that it resembles the letter ' $j$ '. In the dragon, we found that ZII was expressed in alternating sagittal stripes containing ZII+ and ZIIPCs in the posterior cerebellum, similar to what is observed in birds in mammals. More rostrally, all PCs were ZII+, which resembles the pattern of ZII expression in the anterior lobes of birds and mamamls. We suggest that the expression of ZII in the cerebellum as a series of sagittal ZII+ and ZII- stripes is highly conserved but was lost in the snakes in association with limblessness, and lost in the turtles in association with a reduction in axial musculature. Based on this data, we further propose two evolutionary scenarios in which parasagittal stripes evolved in tetrapods: once at the base on all amniotes and subsequently lost in turtle and snakes or independent evolution in squamates, birds and mammals. We conclude with discussing the implications of ZII expression and these two evolutionary scenarios for understanding cerebellar function and organization. 